\title{
The detailed nature of active central cluster galaxies
}

\author{
S. I. Loubser ${ }^{1}$ and I. K. Soechting ${ }^{2}$ \\ ${ }^{1}$ Centre for Space Research, North-West University, Potchefstroom 2520, South Africa \\ email: Ilani.Loubser@nwu.ac.za \\ ${ }^{2}$ Astrophysics, Department of Physics, University of Oxford, Oxford, OX1 3RH, UK \\ email: iks@astro.ox.ac.uk
}

\begin{abstract}
We present detailed integral field unit observations of the central few kiloparsecs of the ionized nebulae surrounding four active central cluster galaxies (CCGs) in cooling flow clusters (Abell 0496, 0780, 1644 and 2052). Our sample consists of CCGs with H $\alpha$ filaments, and have existing data from the X-ray to radio wavelength regimes available, but lacked the detailed optical emission-line (and simultaneous absorption line) data over a broad wavelength range to probe the dominant ionisation processes, excitation sources, morphology and kinematics of the hot gas (as well as the morphology and kinematics of the stars). This, combined with the other multiwavelength data, will form a complete view of the different phases (hot and cold gas and stars) and how they interact in the processes of star formation and feedback detected in central galaxies in cooling flow clusters, as well as the influence of the host cluster. We derive the optical dust extinction maps of the four nebulae. We also derive a range of different kinematic properties, given the small sample size. For Abell 0496 and 0780, we find that the stars and gas are kinematically decoupled, and in the case of Abell 1644 we find that these components are aligned. For Abell 2052, we find that the gaseous components show rotation even though no rotation is apparent in the stellar components. To the degree that our spatial resolution reveals, it appears that all the optical forbidden and hydrogen recombination lines originate in the same gas for all the galaxies. Based on optical diagnostic ratios ([OIII $] \lambda 5007 / \mathrm{H} \beta$ against $[\mathrm{NII}] \lambda 6584 / \mathrm{H} \alpha,[\mathrm{SII}] \lambda 6717,6731 / \mathrm{H} \alpha$, and $[\mathrm{OI}] \lambda 6300 / \mathrm{H} \alpha$ ), all objects contain a LINER nucleus and show extended LINER-like gas emission. We also show that the hardness of the ionizing continuum does not decrease radially within our field-of-view as the emission line ratios do not vary significantly with radius, thus the derived nebular properties are spatially homogeneous. We fit AGN and pAGB stars photoionisation models as well as shock excitation models to our derived diagnostic ratios. We suggest that AGN photoionisation is the most likely ionisation mechanism even though shocks and pAGB stars can not be conclusively eliminated.
\end{abstract}

Keywords. galaxies: formation — galaxies: elliptical and lenticular, $\mathrm{cD}$

We now know that a large fraction of the baryonic mass in massive galaxies is believed to be in diffuse form. Thus a complete view of galaxy formation and evolution necessarily incorporates both the stars and hot gas and an understanding of the processes by which these phases interact. We present detailed IFU observations of the central kiloparsecs of the ionized nebulae surrounding four active CCGs. Our CCGs have $\mathrm{H} \alpha$ filaments, and existing data from the X-ray to radio wavelengths available, to which we now add the detailed optical emission-line (and absorption line) data over a broad wavelength range to probe the dominant ionisation processes, excitation sources, morphology and kinematics of the hot gas (as well as the morphology and kinematics of the stars).

\section{References}

Loubser, S. I., Sánchez-Blázquez, P., Sansom, A. E., \& Soechting, I. K., 2009, MNRAS, 398, 133 